\title{
A rare case of congenital heart disease: anomalous origin of coronary from innominate artery with coronary fistula and truncus arteriosus
}

\author{
wenlei li$^{1}$, Jianbin $\mathrm{Li}^{1}$, Li Ma ${ }^{1}$, Weidan $\mathrm{Chen}^{1}$, Minghui Zou ${ }^{1}$, and Xinxin $\mathrm{Chen}^{1}$ \\ ${ }^{1}$ Guangzhou Women and Children's Medical Center
}

November 9, 2020

\begin{abstract}
Anomalous origin of a single coronary artery arising from the innominate artery associated with coronary artery fistula and truncus arteriosus is an extremely rare anomaly. A 16-month-old girl received Rastelli procedure during in the surgical treatment of truncus arteriosus. We found the coronary artery anomaly in the operation. The operation went well, and the patient is doing well without any respiratory symptoms or active intolerance 28 months postoperatively.There may have a connection between a single coronary artery arising from the innominate artery and truncus arteriosus according to the literature reports. The three defects described above are rarely found together, indeed, such a case as ours may be the first reported in the literature.
\end{abstract}

Title: A rare case of congenital heart disease: anomalous origin of coronary from innominate artery with coronary fistula and truncus arteriosus

Wenlei Li, M.D ${ }^{1}$., Jianbin Li, M.D. ${ }^{1}$, Li Ma, M.D. ${ }^{1}$, Weidan Chen, M.D. ${ }^{1}$, Minghui Zou, M.D. ${ }^{1}$, Xinxin Chen, M.D. ${ }^{1 *}$

${ }^{1}$ Department of Cardiac Surgery, Guangzhou Women and Children's Medical Center, Guangzhou Medical University, Guangzhou, China

Running Title: Coronary origin from innominate artery

*Corresponding Author

Xinxin Chen, M.D.

Department of Cardiac Surgery

Guangzhou Women and Children's Medical Center, Guangzhou Medical University

9\# Jinsui Road, Guangzhou 510623 (China)

Tel: +862038076180

E-Mail: zingerchen@163.com

Keywords: anomalous origin of coronary; coronary fistula; truncus arteriosus

Abstract

Anomalous origin of a single coronary artery arising from the innominate artery associated with coronary artery fistula and truncus arteriosus is an extremely rare anomaly. A 16-month-old girl received Rastelli procedure during in the surgical treatment of truncus arteriosus. We found the coronary artery anomaly in 
the operation. The operation went well, and the patient is doing well without any respiratory symptoms or active intolerance 28 months postoperatively. There may have a connection between a single coronary artery arising from the innominate artery and truncus arteriosus according to the literature reports. The three defects described above are rarely found together, indeed, such a case as ours may be the first reported in the literature.

\section{Introduction}

Anomalous origin of a single coronary artery arising from the innominate artery associated with coronary artery fistula and truncus arteriosus is an extremely rare anomaly. We report this successful surgical treatment of this anomaly in an infant in whom the morphology was found in the operation.

\section{Case Report}

In May 2018, a 16-month-old girl was charged in our hospital because of heart murmur. The chest radiogram showed cardiomegaly and prominent increased pulmonary vascularity. The electro-cardiogram showed biventricular hypertrophy and q-wave in lead I, avL, V5, V6. Our echocardiogram found truncus arteriosus (Type I, Collett and Edwards) with severe pulmonary hypertension, the truncal valve has 3 leaflets with no stenosis or regurgitation. We measured pulmonary artery pressure was 69/51(60) $\mathrm{mmHg}$, pulmonary vascular resistance was 12.87 wood $\mathrm{U} / \mathrm{m}^{2}$, and $65 / 50(60) \mathrm{mmHg}, 3.49$ wood $\mathrm{U} / \mathrm{m}^{2}$ after oxygen inhalation, respectively, by performing cardiac catheterization as the patient is older than 6 months. No significantly surgical contraindications were found in preoperative evaluation.

An operation was performed with the plan of Rastelli procedure. However, after a median sternotomy, we found there was no coronary arose from the aorta, but a single artery arose from innominate artery and branched into left and right coronary artery. The distribution was like that of either a right or a left normal coronary artery. The cardioplegia infusion needle was inserted in the innominate artery near the ostium of this single coronary artery, the proximal and distal of the innominate artery was blocked during the cardioplegia infusion. (shown in Fig. 1) The aorta was cross-clamped and infusion of HTK solution at $4^{\circ} \mathrm{C}$ was antegraded through the infusion needle. During the infusion, we found some HTK solution leaked from the ventricles. After the ascending aorta was transected, no coronary artery arose from the truncus, and we found a coronary fistula below the non-coronary aortic sinus, which is an uncommon anomalous communication between the coronary artery and left ventricle. After fistula closed, the cardiac arrest was obtained successful.

The pulmonary branch arteries were then disconnected from the truncus and a $16 \mathrm{~mm}$ bovine jugular vein conduit was placed from the right ventricle to the pulmonary branch arteries. Pulmonary artery flow study was performed by using a flow rate to the pulmonary arteries of $3 \mathrm{~L} / \mathrm{min} / \mathrm{m}^{2}$ with a maximum mean pulmonary artery pressure of $23 \mathrm{~mm} \mathrm{Hg}$. A patch was used to close the ventricular defect. Partial closure of atrial septal defect with $4 \mathrm{~mm}$ residual shunt. The cardiopulmonary bypass (CPB) and cross-clamp times were 196 and 113 minutes, respectively. The sternum was closed after weaning from CPB with support from inotropic agents, dopamine, and epinephrine. The intubation time was 4 days and after using of 1 day humidified high flow nasal cannula oxygen therapy, the patient left the ICU at post-op day 8 and discharged from hospital at post-op day 15 . The echocardiogram showed satisfactory intracardiac correction with moderate mitral and tricuspid valve regurgitation, and atrial level two-way shunt associated with residual pulmonary hypertension.

At this writing, the patient is doing well without any respiratory symptoms or active intolerance 28 months postoperatively. The latest echocardiogram showed $3 \mathrm{~mm}$ left-right shunt of atrial level and mild mitral and tricuspid and pulmonary valve regurgitation. No pulmonary artery stenosis or dilation was observed. The $\mathrm{N}$-terminal pro-brain natriuretic peptide concentration was $220.14 \mathrm{pg} / \mathrm{ml}(<500 \mathrm{pg} / \mathrm{mL})$. This case report has received approval from the institutional ethics committee of the Guangzhou women and children's hospital.

\section{Discussion}

Truncus arteriosus also known as common arterial trunk, is an uncommon (1-3\% of all cases of congenital 
heart disease) congenital cardiac defect that presents at birth. ${ }^{1}$ It supplies blood to the systemic and pulmonary circulation simultaneously, leading to pulmonary circulation increased and congestive heart failure. Within a few weeks after birth, the pulmonary hypertension will significantly increase even leading pulmonary hypertension crisis after operation. Mayo Clinic's early experience also showed that early mortality was associated with increased pulmonary vascular resistance. Currently, the surgical treatment of Truncus arteriosus is generally advocated in neonates or within three months after birth to reduce the incidence of pulmonary hypertension crisis. ${ }^{2}$ In this case, the patient was 16 -month-old, so the cardiac catheterization was performed to exclude the surgical contraindication.

Anomalies of the coronary arteries found incidentally in $0.8-1.3 \%$ of healthy individuals ${ }^{3}$, including anomalous origin, anomalous course and anomalous termination. ${ }^{4}$ The origin of a single coronary artery outside aortic sinuses or above the pulmonary valve cusps is extremely uncommon. Mayer, in $1827^{5}$, described a case where the vessel arose form the right carotid artery. In 1956 Cree ${ }^{6}$ reported a single coronary artery arose from the innominate artery and divided into a right coronary artery and a left marginal artery, in a patient with single ventricle and truncus arteriosus. Keeling in $1970^{7}$ and Zachariah and Reif in $1974{ }^{8}$ both reported cases of a single coronary artery arising from the innominate artery with additional truncus arteriosus and single ventricle malformations. In each instance, the anomaly diagnosis was made at necropsy. Liu et al ${ }^{9}$ in 2010 reported case of a 42-day-old boy with anomalous origin of a single coronary artery from the right branchiocephalic trunk associated with hypoplastic left ventricle, mitral atresia, truncus arteriosus. Due to the poor prognosis and challenging surgery, the boy was not received operation.

In our case, a single coronary arising from a much higher site on the innominate artery. It would have been hard to perform coronary artery bypass grafting for such a small infant. Although we performed preoperative cardiac catheterization for this patient, the anomalies of the coronary arteries were not be found as coronary angiography is too dangerous to be perform on infant. Lacking picture of computed tomographic angiography for this special patient is a regret. Due to the existing coronary artery fistula, the cardiac arrest could not be obtained successfully. It is important to select the location where cardioplegia infusion needle inserts. Careful observation is needed when cardioplegia solution infusing.

There may have a connection between a single coronary artery arising from the innominate artery and truncus arteriosus according to the literature reports. The cardiac surgeon must be aware of the abnormal anatomy to avoid accidental ligation or cross-clamping or transection the anomaly coronary artery during surgery. The three defects described above are rarely found together, and we performed successful surgical treatment; indeed, such a case as ours may be the first reported in the literature. Statements

Acknowledgement

None.

\section{Statement of Ethics}

This case report has received approval from the institutional ethics committee of the Guangzhou women and children's hospital.

Conflict of Interest Statement

The authors have no conflicts of interest to declare.

Funding Sources

This work was supported by the National Natural Science Foundation of China (grant number 81800280).

Author Contributions

Wenlei Li writing the article and obtaining funding;

Jianbin Li made drawing and data acquisition;

Li Ma did data acquisition and critical revision of the article; 
Weidan Chen provided advice about study protocol;

Minghui Zou did data acquisition and contributed to manuscript preparation;

Xinxin Chen designed this study, critical revision of the article. References

1. Russell HM, Pasquali SK, Jacobs JP, et al. Outcomes of repair of common arterial trunk with truncal valve surgery: a review of the society of thoracic surgeons congenital heart surgery database. Ann Thorac Surg. 2012;93(1):164-169; discussion 169.

2. Marcelletti C, McGoon DC, Mair DD. The natural history of truncus arteriosus. Circulation. 1976;54(1):108-111.

3. Angelini P, Velasco JA, Flamm S. Coronary anomalies: incidence, pathophysiology, and clinical relevance. Circulation. 2002;105(20):2449-2454.

4. Greenberg MA, Fish BG, Spindola-Franco H. Congenital anomalies of the coronary arteries. Classification and significance. Radiol Clin North Am. 1989;27(6):1127-1146.

5. Mayer SF. Eine Missgeburt mit einem Parasiten auf der Brust. J Chir Augen Heilk. 1827;10:44.

6. Cree IC. Truncus arteriosus and a single ventricle. Br Heart J. 1956;18(4):553-556.

7. Keeling JW. Anomalous origin of a single coronary artery. J Pathol. 1970;102(3):174-177.

8. Zachariah S, Reif R. Anomalous origin of single coronary artery with multiple heart malformations. $\mathrm{Br}$ Heart J. 1974;36(11):1144-1145.

9. Liu F, Huang G, Zhang J. Anomalous origin of a coronary artery from the right branchiocephalic trunk associated with complex congenital heart disease. Pediatr Cardiol. 2010;31(1):163-165.

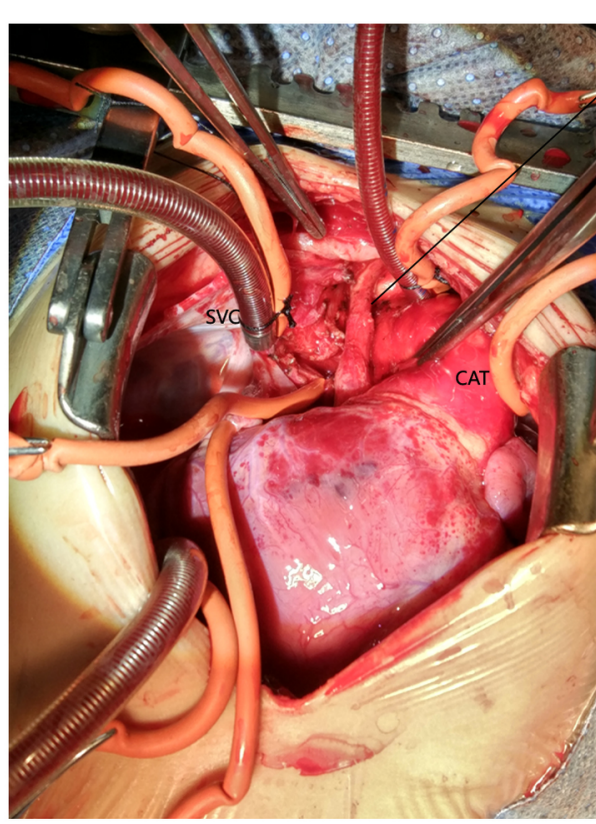

(a)

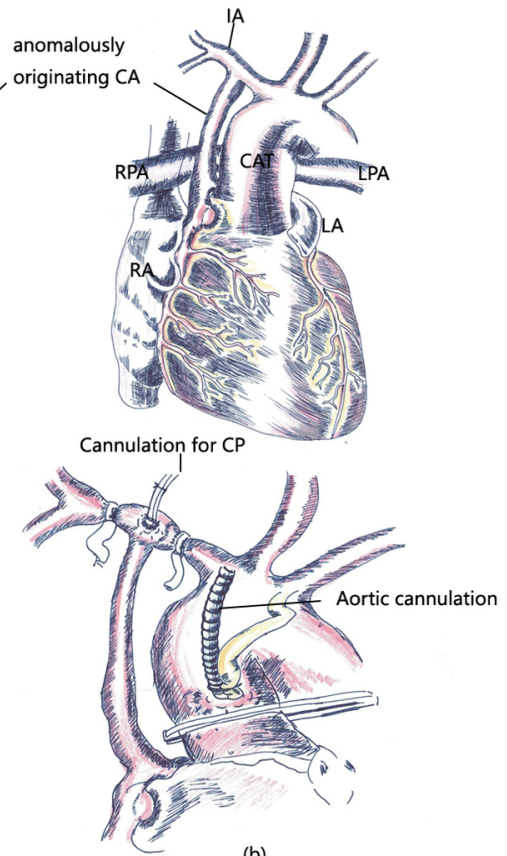

(b) 